\title{
Review
}

\section{Temporomandibular disorders and dental occlusion. A systematic review of association studies: end of an era?}

2 D. MANFREDINI id, L. LOMBARDO \& G. SICILIANI Post-graduate School in Orthodontics, University

3 of Ferrara, Ferrara, Italy

SUMMARY To answer a clinical research question: 'is there any association between features of dental occlusion and temporomandibular disorders (TMD)?' A systematic literature review was performed. Inclusion was based on: (i) the type of study, viz., clinical studies on adults assessing the association between TMD (e.g., signs, symptoms, specific diagnoses) and features of dental occlusion by means of single or multiple variable analysis, and (ii) their internal validity, viz., use of clinical assessment approaches to TMD diagnosis. The search accounted for 25 papers included in the review, 10 of which with multiple variable analysis. Quality assessment showed some possible shortcomings, mainly related with the unspecified representativeness of study populations. Seventeen $(N=17)$ articles compared TMD patients with nonTMD individuals, whilst eight papers compared the features of dental occlusion in individuals with TMD signs/symptoms and healthy subjects in non-patient populations. Findings are quite consistent towards a lack of clinically relevant association between TMD and dental occlusion. Only two (i.e., centric relation [CR]-maximum intercuspation [MI] slide and mediotrusive interferences) of the almost forty occlusion features evaluated in the various studies were associated with TMD in the majority (e.g., at least $50 \%$ ) of single variable analyses in patient populations. Only mediotrusive interferences are associated with TMD in the majority of multiple variable analyses. Such association does not imply a causal relationship and may even have opposite implications than commonly believed (i.e., interferences being the result, and not the cause, of TMD). Findings support the absence of a diseasespecific association. Based on that, there seems to lack ground to further hypothesise a role for dental occlusion in the pathophysiology of TMD. Clinicians are encouraged to abandon the old gnathological paradigm in TMD practice.

KEYWORDS: dental occlusion, temporomandibular disorders, association, systematic review

Accepted for publication 6 June 2017

\section{Introduction}

The relationship between dental occlusion and temporomandibular disorders (TMD) is still a controversial topic in dentistry. Indeed, whilst communities of oro-facial pain experts seem to have embraced a biopsychosocial model of TMD (1), within the broader context of oro-facial pain conditions (2), professionals focusing on the study and restoration of dental occlusion (i.e., orthodontists, prosthodontists, restorative dentists) are historically less prone to accept concepts that diminish the importance of occlusal dogmas (3). Thus, the occlusion-TMD field is still often source of speculations.

Temporomandibular disorders are a heterogeneous group of conditions affecting the temporomandibular joints (TMJ), the jaw muscles and/or the related structures (4). Their prevalence is not negligible at the general population level (5), and patient populations are characterised by relevant psychosocial 
impairment, which is often unrelated to the physical diagnosis (6).

Dental occlusion is the core of dentistry. Decades of researches have progressively shed light to many issues concerning the management of occlusion in the clinical practice (7). A purported causal relationship between 'malocclusion' and TMDs has been advocated for years by the precepts of gnathology (8), but the occlusal paradigm for TMD has never been convincingly validated (9). Observations that conservative management of TMD symptoms is almost always enough to achieve positive outcomes (10), and that chronic pain subjects are individuals with specific personality, and not occlusal, profiles (11-13), support the concept of neutrality as far as the effects of occlusal therapies on TMDs are concerned (14-16).

Notwithstanding, this did not reduce the impact of occlusion-related issues in the field of TMD and orofacial pain, as easily perceivable with a look at popular information channels. Thus, a gap still exists between the research clinicians and the communities of dental practitioners. A possible explanation is that the association between dental occlusion, and TMDs have never been reviewed systematically. The available knowledge is mainly based on seminal papers and comprehensive reviews, which suggest clinicians to focus on other factors than dental occlusion to manage effectively TMD patients but failed to provide an end point to the gnathological era (17-19). Indeed, on the other hand, the absence of a systematic approach to literature assessment so far may justify some controversial claims that the 'occlusal question' is still unsolved $(20,21)$.

Based on these premises, this manuscript attempts to review the literature to answer a clinical research question: 'Is there any association between features of dental occlusion and temporomandibular disorders?'

\section{Materials and methods}

\section{Search strategy}

On 18th January 2017, a systematic search in the medical literature was performed to identify all peerreviewed English language papers that were relevant to the review's aim. As a first step, a search query 'dental occlusion (MeSH term)' AND 'temporomandibular joint disorders (MeSH term)' were performed within the National Library of Medicine's Medline database to retrieve a list of potentially relevant papers. Limits were set to English language studies on humans, with an available abstract. Based on title and abstract (TiAb) assessment, the studies were selected for full-text retrieval and potential inclusion independently by two of the authors (D.M, L.L.), who also performed data extraction by consensus decision. Both authors contributed to the search expansion by checking for additional papers in the Scopus and Google Scholar databases, in the reference lists of potentially relevant papers, and in their own personal and institutional libraries.

The criteria for admittance in the systematic review were based on: (i) the type of study, viz., clinical studies on human adult populations assessing the association between temporomandibular disorders (e.g., signs, symptoms, specific diagnoses) and features of dental occlusion by means of single or multiple variable analysis, and (ii) their internal validity, viz., use of validated clinical or radiological assessment approaches to TMD diagnosis. Investigations with case-control design (selected populations of TMD patients versus non-TMD individuals) as well as studies assessing the TMD-dental occlusion association at the general population level (i.e., non-patient populations) were both included. Studies on self-reported TMD diagnosis and/or unclear protocols to assess occlusal features were excluded.

\section{Systematic assessment of papers}

The methodological characteristics of the selected papers were assessed based on a format that enabled a structured summary of the articles in relation to four main issues, viz., ' $\mathrm{P}$ ' - patients/problem/population, ' $\mathrm{I}$ ' - intervention, ' $\mathrm{C}$ ' - comparison and ' $\mathrm{O}$ ' - outcome (PICO), for each of which specific questions were constructed (22).

For each article, the study population (' $\mathrm{P}^{\prime}$ ) was described based on the criteria for inclusion and the demographic features of the non-TMD individuals. The study design was described in the section reserved to questions on the study intervention (' $\mathrm{I}$ '), and information was gathered on the type and number of occlusal features under investigation. The comparison criterion ('C') was based on the description of the TMD patients. The study outcome ('O') was evaluated in relation to the measures of association between the assessed occlusal features and TMD, either with single or multiple variable analyses. 
Quality assessment

Critical appraisal of studies included in the review was performed based on the Newcastle-Ottawa Scale (NOS) for case-controls studies. NOS assesses the quality of reviewed studies by evaluating eight items concerning the Selection, Comparability and Exposure categories.

The Selection category consists of four items: case definition, representativeness of cases, selection of controls and definition of controls. In this review, case definition was considered adequate when cases (i.e., TMD patients) were identified with clinical and/or imaging assessment for TMD status; case representativeness was judged positively when they were recruited consecutively; selection of controls was endorsed for community samples; definition of controls was considered adequate when they had no history and no current presence of TMD signs and/or symptoms.

The Comparability category is made of a single item evaluating the comparability of cases and controls based on the design or analysis. The study was endorsed positively if it controls for dental occlusal variables by adopting a multiple variable design and/ or it controls for additional factors (e.g., bruxism, psychosocial factors).

The exposure category consists of three items, assessing the ascertainment of exposure (i.e., dental occlusion features), the use of the same method of ascertainment for cases and controls, and the nonresponse rate. Ascertainment was considered adequate if the assessment of dental occlusion was based on clinical examination and/or evaluation of dental casts, for both cases and controls. The non-response rate item was endorsed positively when it was clearly specified the number of non-respondent individuals with respect to the total of invited/recruitable people.

Based on the above, a study can be awarded a maximum of one star for each item within the Selection and Exposure categories. A maximum of two stars can be given for Comparability. Thus, the highest quality studies are assigned a score of 9.

\section{Results}

Search results

The search allowed identifying 1670 citations in the Medline database, 848 of which were excluded when search limits were applied. Thus, 822 citations were screened for eligibility. As shown in Fig. 1, after excluding the citations that were clearly not pertinent for the review's aim based on their title and abstract, 46 papers were retrieved in full text and were assessed to reach consensus as to include/exclude the

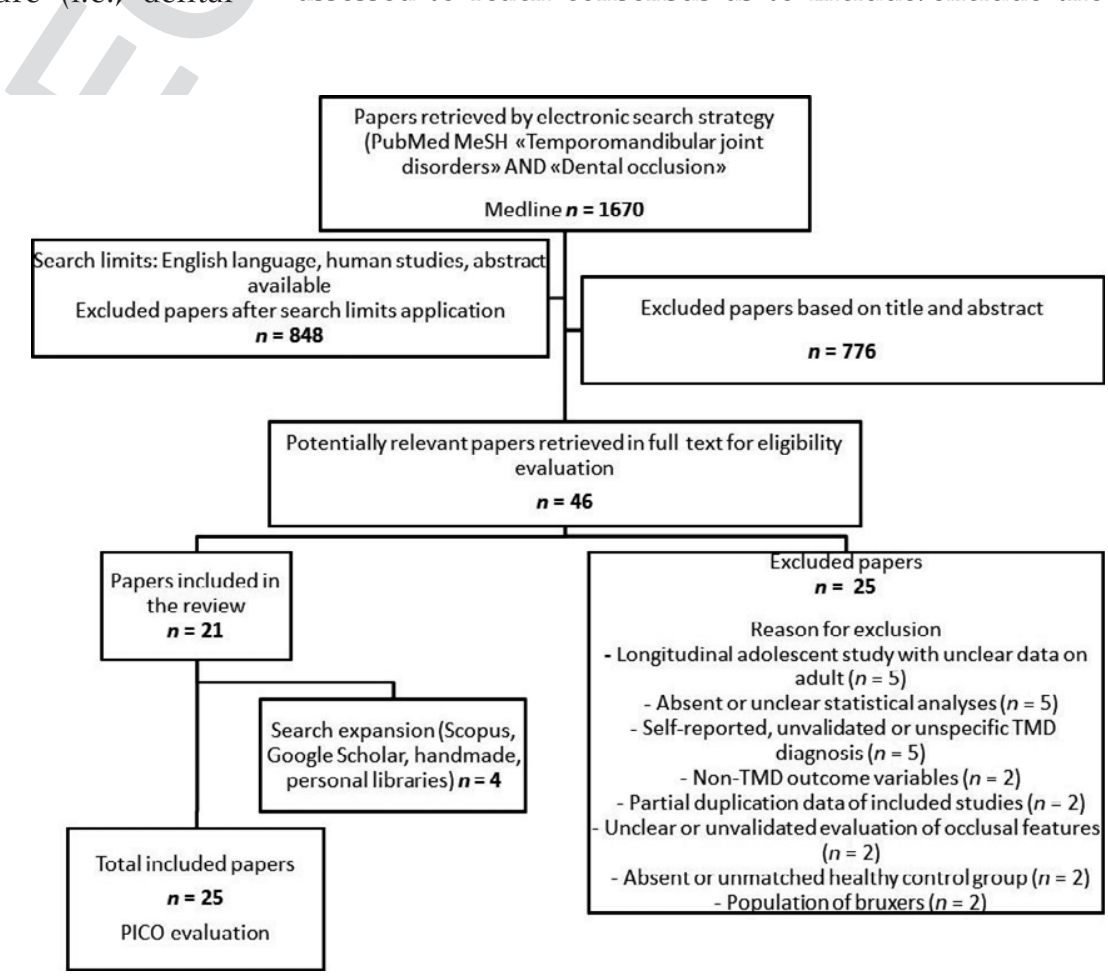

Fig. 1. Flow chart of search strategy and results. 
papers for/from systematic assessment. Consensus decision was to exclude 25 of the 46 papers. Reasons for exclusion were described in Table 1. Search expansion strategies allowed including four additional papers, thus accounting for a total of 25 papers included in the review (48-72).

Table 1. Excluded papers after full-text reading

Study first author,

year

Reason for exclusion

Main finding(s) - TMD/occlusion

Egermark, 1987 (23) Longitudinal study on adolescents, unclear data on adulthood

Runge, 1989 (24)

No statistical analysis

Unilateral contact in ICP at 20 years associated with TMJ sounds

Dental occlusion features do not seem related with TMJ click

Al-Hadi, 1993 (25) No validated TMD criteria, unclear statistical analysis Single variable: association between Class II- 1 and TMD

Christensen, 1996 (26) Unclear 'TMD' criteria (click sound?), no statistical analysis

Similar prevalence of mediotrusive tooth guidance in subjects with and without joint sounds

Donegan, 1996 (27) Unclear 'TMD' criteria (click sound?), no statistical analysis

Similar prevalence of canine guidance in subjects with and without joint sounds

Liu, 1997 (28)

Sample containing children and adolescents, unclear data on adulthood

No association between morphologic occlusion and TMD

Minagi, 1997 (29)

Study on TMJ dynamics

Not pertinent

Ciancaglini, 1999(30) Study on occlusal support, no validated TMD criteria

Multiple variable: stiffness of the jaw associated with loss of occlusal support

Pullinger, 2000 (31) Combined sample of included 1993 and 2006 studies

Significant relative risk for disease (odds ratio $>2: 1$ ) was mainly associated with infrequent, more extreme ranges of occlusion measurements.

John, 2002(32)

Fuji, 2003 (33)

Sarita, 2003 (34)

Pahkala, 2004 (35)

Mundt, 2005 (36)

Sipila, 2006 (37)

Wang, 2007 (38)

Badel, 2008 (39)

Wang, 2009 (40)

Marklund, 2010 (41)

Self-report TMD assessment No measure of association

Study on chewing ability and shortened dental arch Study on adolescents, unclear data on adulthood Subsample of Gesch et al., 2004

Patients with unspecified facial pain

Sample of patients with tightly locked occlusion, diagnosed with unspecific criteria

Unmatched age of disc displacement ( 35.3 years) and control (23.4 years) groups, unclear criteria for measuring occlusal vertical dimension (OVD)

Sample of patients with missing posterior teeth (no controls without missing posterior teeth), generic TMD diagnosis

Longitudinal study on students (non-patients), unspecific TMD/occlusion relationship

Lauriti, 2013 (42) Study on adolescents

Manfredini, 2014 (43) Study on bruxers

Manfredini, 2014 (44) Study on the role of extreme occlusal features in bruxers

Manfredini, 2014 (45) Study on TMD patients, no control group

Baldini, $2015(46) \quad$ Study on occlusion time

Michelotti, 2016 (47) Longitudinal study, no standardised evaluation
No association with overbite and overjet Interferences more frequent in the side of pain and clickling

Not pertinent

Not pertinent

See main paper

No relationship between TMD and occlusal variables Single variable: association with unspecified TMD

Single variable: association of TMD with reduced OVD and uneven dental contacts, no association with overjet, overbite, non-centred incisor midline Multiple variable: number of quadrants with missing posterior teeth associated with TMD

Not pertinent

No association with Angle class, open bite, cross-bite Multiple variable: only one significant (molar asymmetry) of 11 occlusal features

Not pertinent

No association between TMJ click and seven occlusal variables

Clinically unrelevant differences between TMD and TMD-free

Single variable: association between click and crossbite independent on cross-bite correction 


\section{Study findings}

Seventeen $(N=17)$ of the included studies had a case-control design, comparing a population of TMD patients with non-TMD individuals, whilst eight papers compared the features of dental occlusion in individuals with TMD signs/symptoms and healthy subjects in non-patient populations. Structured reading of the included articles showed a high variability as far as the occlusal features under evaluation and the TMD diagnosis (i.e., muscle, joint or combined disorders) are concerned. Anterior vertical (i.e., overbite) and horizontal overlap (i.e., overjet) and slide from centric relation (CR) to maximum intercuspation (MI) were the most frequently investigated occlusal features. Multiple variable analysis was performed only in 10 papers, whilst the other investigations provide an evaluation of the association between TMD and some selected occlusal features by means of single variable analysis. Given the heterogeneity of study designs, meta-analysis of data or quality assessment could not be performed. Methodological features and main findings concerning the possible association between dental occlusion and temporomandibular disorders in patients and non-patient populations are summarised in Tables 2 and 3.

In summary, the pattern of described association is quite consistent across studies towards a lack of clinically relevant association between TMD and dental occlusion. Only two (i.e., CR-MI slide and mediotrusive interferences) of the almost forty dental occlusion features that have been evaluated in the different studies are associated with TMD in the majority (i.e., at least $50 \%$ ) of single variable analyses in patient populations, and only mediotrusive interferences are associated with TMD in the majority of multiple variable analyses, with an OR of 2.45 for myofascial pain (57) and 2.14 for disc displacement (64). Other potential clinically relevant odds ratio (OR) for TMD (i.e., higher than 2) in multiple variable analysis are reported occasionally. Summary of findings per each of the most frequently investigated occlusal feature is reported in Table 4.

\section{Quality assessment}

Of the 25 papers included in the review, only two received an 8-star score. The majority of papers felt within the 4- to 6-star range. The most common shortcomings were the unspecified representativeness of the cases and unclear non-response rate. Thus, when considering the quality of the selected articles, the assessment showed the moderate level of the reviewed articles as well as their qualitative homogeneity. However, their methodological heterogeneity prevented a meta-analysis of data (Table 5).

\section{Discussion}

For years, the focus of dental professionals approaching patients with temporomandibular disorders has been solely based on the assessment and correction of purported abnormalities of dental occlusion (7). Over the past few decades, emerging evidence has grown in support of a biopsychosocial model of TMD pain (73). Notwithstanding that, it seems that the new paradigm diminishing the role of occlusal factors has not been fully accepted by some dental clinicians. There are several possible explanations for this resistance.

First, the dental profession has historically played a primary role as the caregivers for TMD patients. In addition, financial disincentives associated with the reduced importance of dental occlusion as well as patients' expectations to receive a dentally oriented treatment contribute to limit the acceptance of other concepts and practices. Finally, clinical observations of paradox effectiveness of seemingly occlusally oriented therapies (e.g., oral appliances) have persuaded many clinicians to continue using those approaches. Such difficulties can be easily appraised by browsing the Internet and giving a look at the number of congresses, events and technological devices that still focus on the search for an ideal occlusion in 'dysfunctional' patients. Speculative theories on the relationship between body posture and occlusal abnormalities, which have been refuted by all reviews on the topic $(18,74)$, best exemplify the situation. On the other hand, a definitive summary of the relationship between TMD and dental occlusion has not been provided so far. The heterogeneity of literature as far as the study designs and research methods is concerned may explain why most current state of the art reviews are more narrative than systematic (17).

A cause-and-effect relationship between two phenomena can be hypothesised with the accomplishment of a set of criteria for causality (e.g., strong and consistent association; temporality; theoretical and 


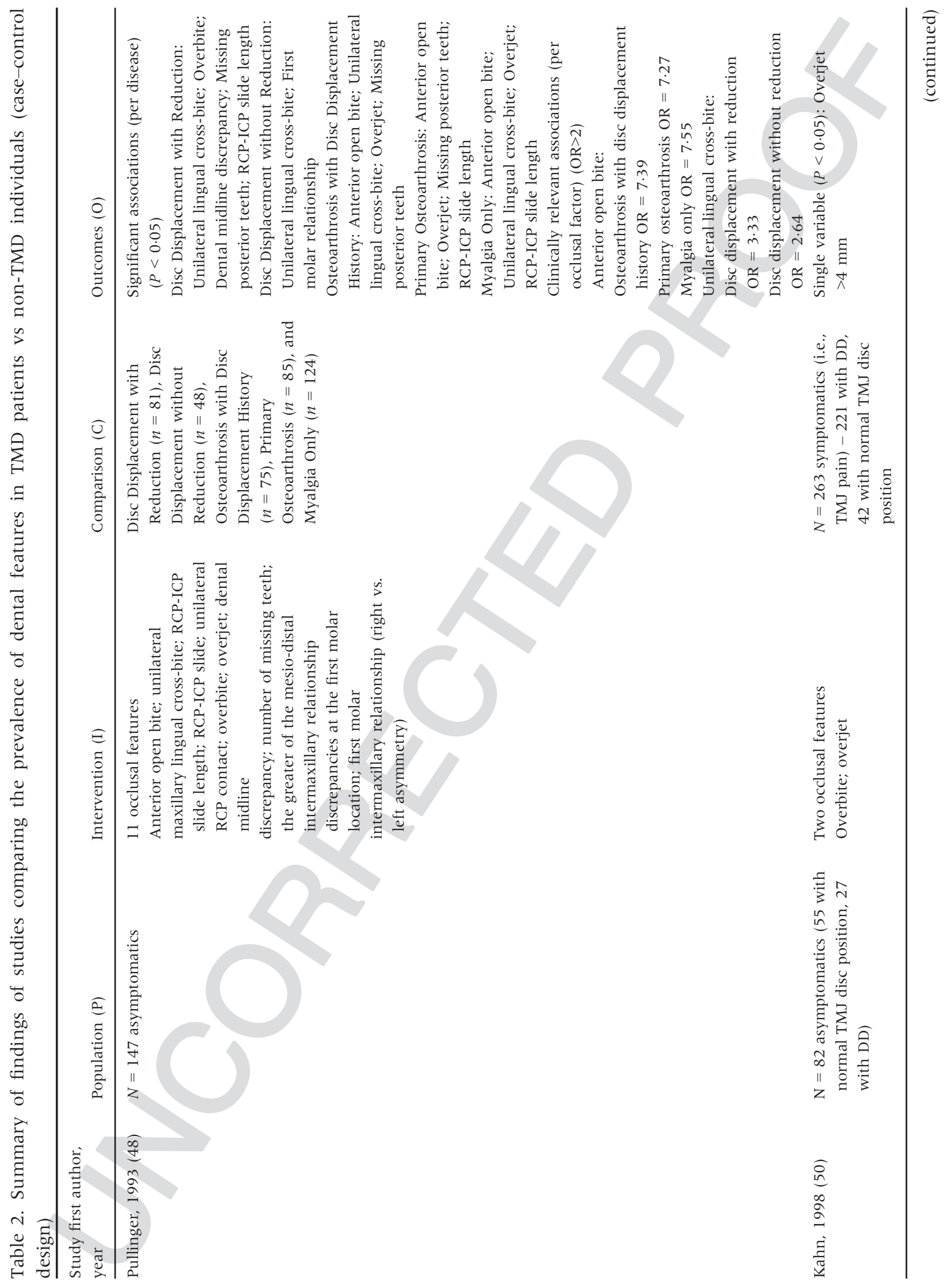




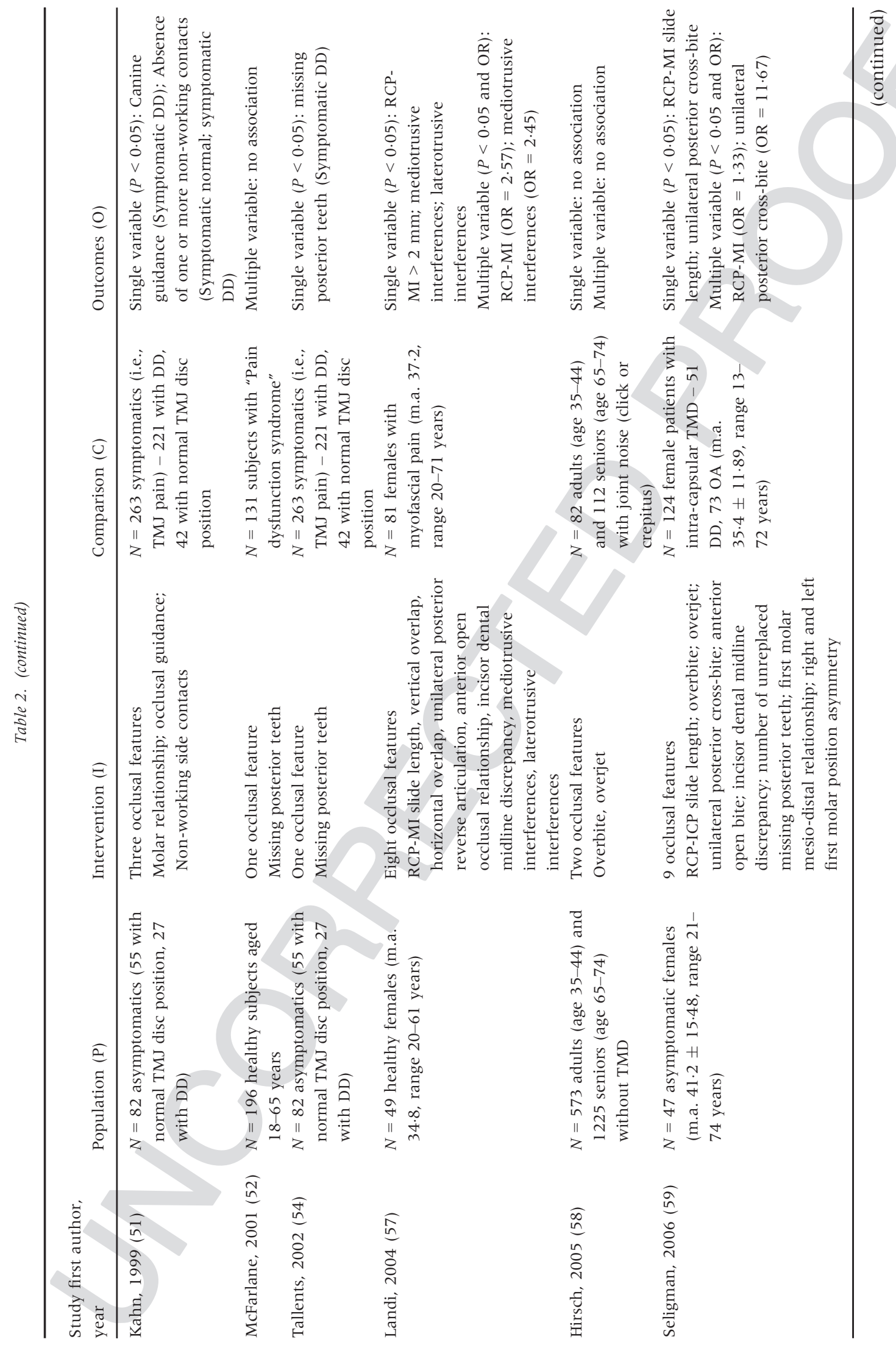

(C) 2017 John Wiley \& Sons Ltd 


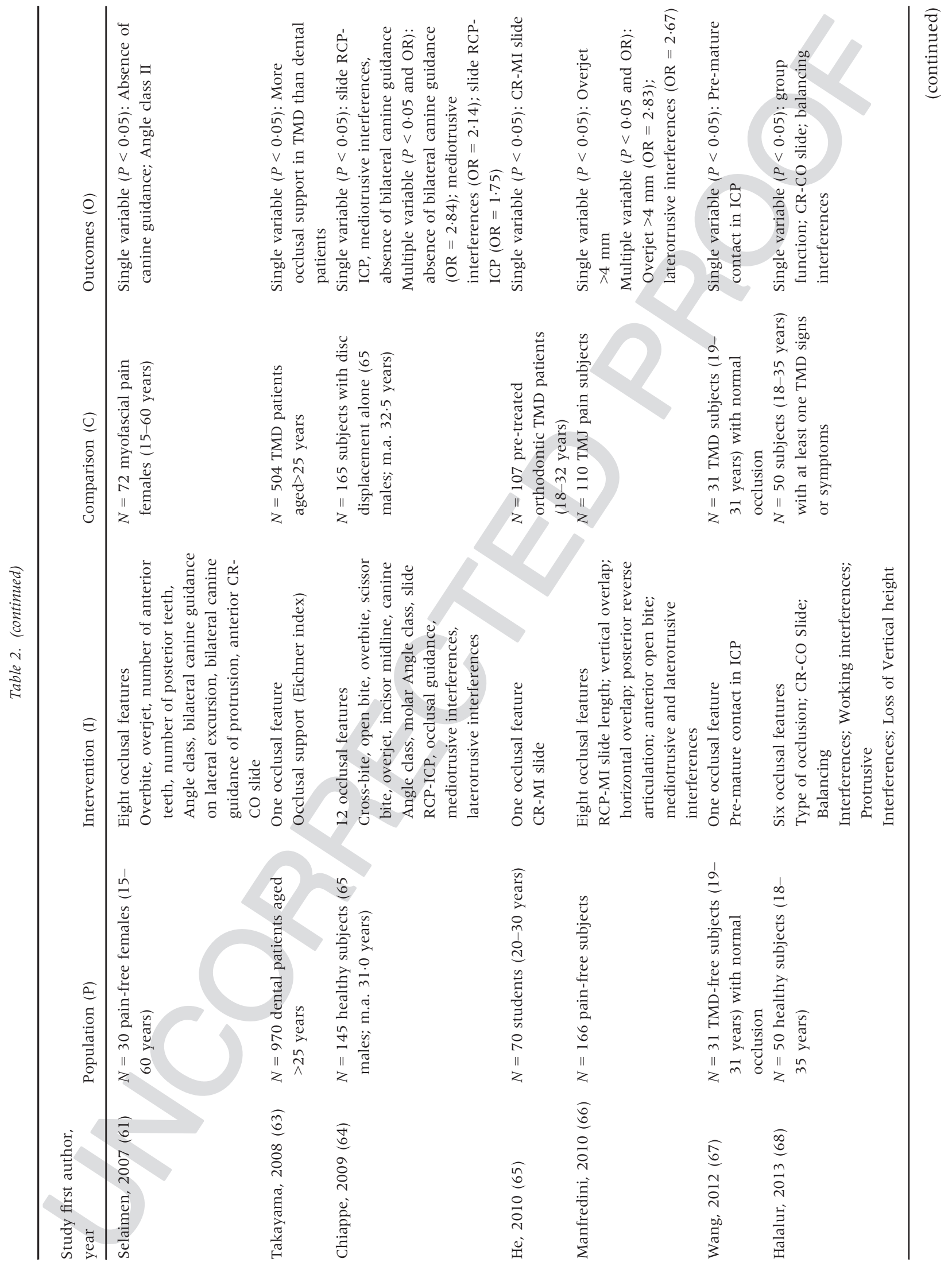




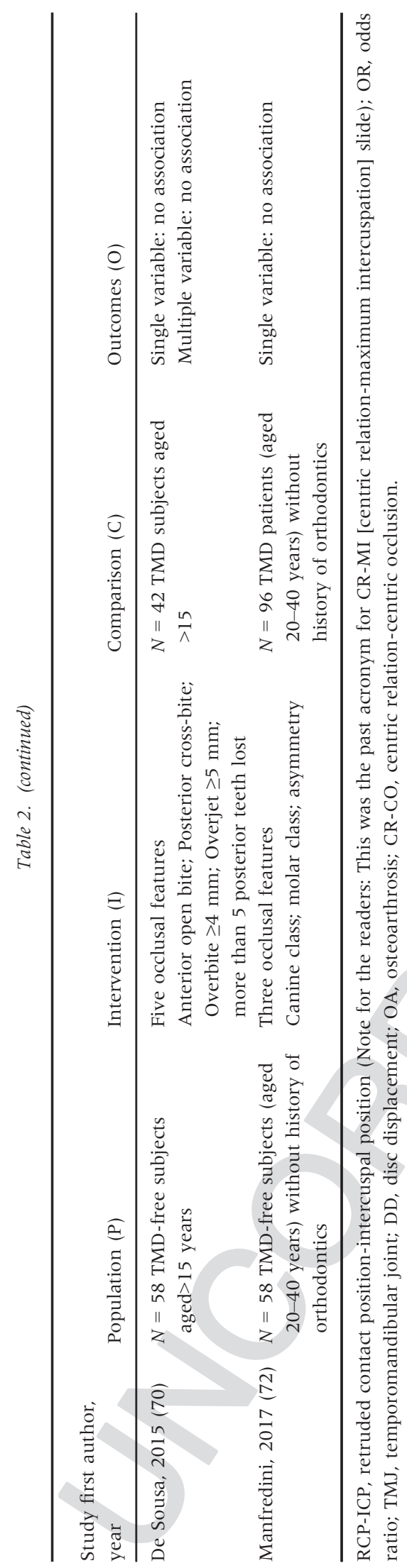

experimental validity; dose-response relationship; specificity, coherence and analogy with available knowledge) (75). Among those criteria, the presence of an association between the two conditions (i.e., the purported causal factor [dental 'malocclusion'\} should be significantly more frequent in diseased ['TMD'] than healthy subjects, as well as diseased individuals should have a higher frequency of the purported causal factor than its absence) is the basic pre-requisite to get deeper into the assessment of causal hypothesis. This manuscript has systematically reviewed the literature on the topic, by including all papers that may be pertinent for the assessment of the association between dental occlusion features and TMD, on the premise that such associations are the first requirement for even considering a causal relationship between them.

Findings of this reviews support the absence of consistent, clinically relevant associations between TMD and the various features of dental occlusion. Reported associations were scarce, weak and mainly drawn from studies with a single-variable design. Multiple variable analyses described associations that reached strength for possible clinical relevance only in a few papers on patient $(48,57,59,64,66)$ or non-patient populations $(56,60)$. Each of those papers identified no more than two occlusal variables in association with TMD among the full spectrum of features under investigation (i.e., ranging from 6 to 33). Conversely, each of those variables was not associated with TMD in more than a single paper. In short, patterns of association are not consistent across studies and may even be due to chance. Thus, the absence of the fundamental pre-requisite of association between the two phenomena leads to conclude that a causal role for dental occlusion in temporomandibular disorders should not be hypothesised.

Such findings may offer some interesting arguments for discussion. First, there is a scarce literature on the topic, and the quality of reviewed articles was, on average, less than optimal. Such finding contrasts with the number of papers on the different strategies to correct purported abnormalities of dental occlusion by means of orthodontics or prosthodontic treatments and calls into question the ethical principles of medicine $(76,77)$. Second, there is a wide methodological variability between the different investigations as for the assessed TMD signs and symptoms. The studies adopting multiple variable models, which best depict 


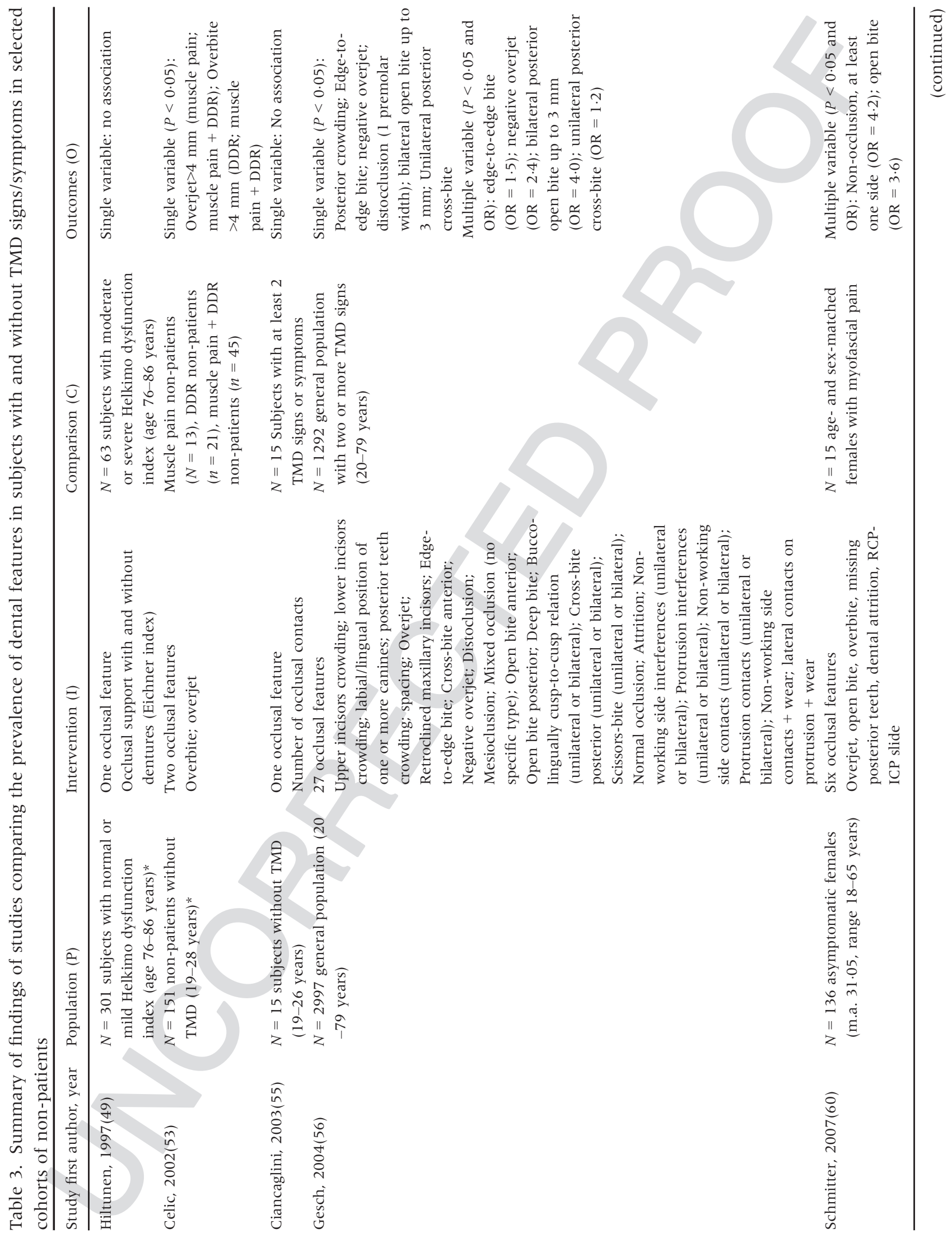




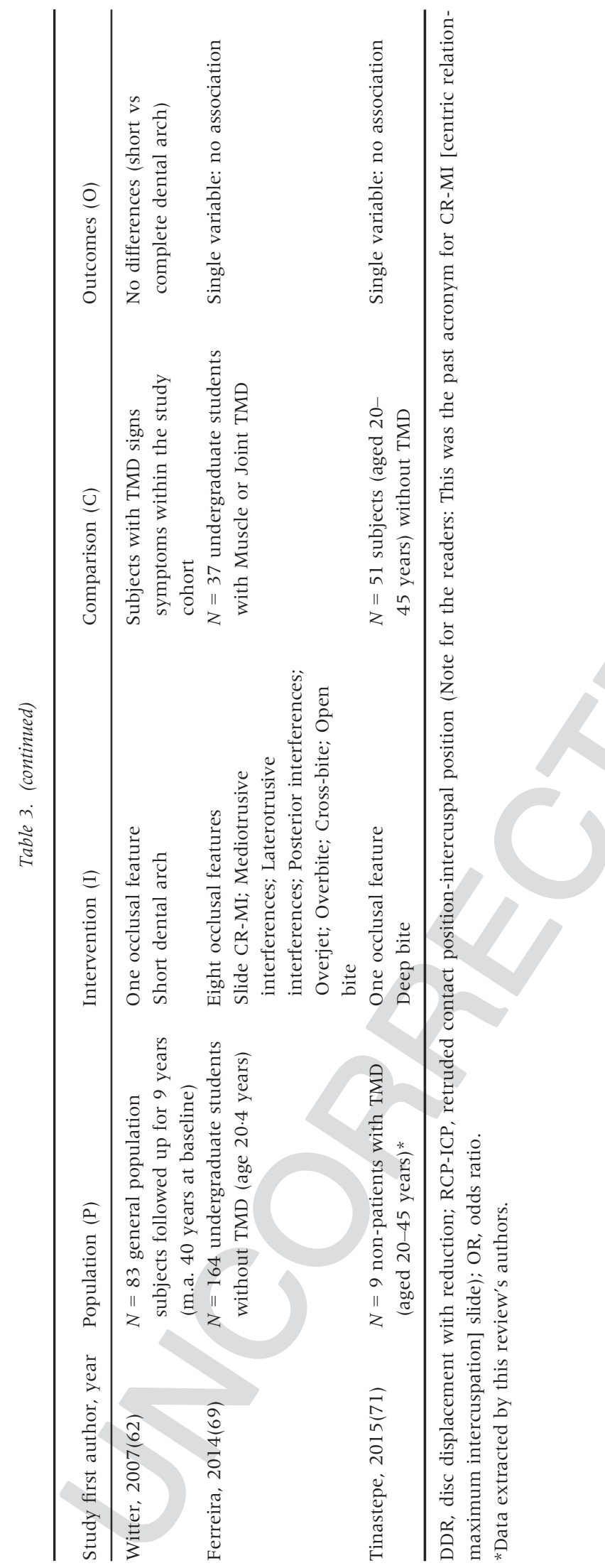

the biological system, comprehend a very wide range of morphological and functional occlusal variables. Such a variability of contents makes meta-analysis of findings not possible and limits the generalisation of quality assessment. Despite that, it should be borne in mind that studies performing a single variable assessment of the TMD-occlusion assessment as well as those recruiting general population subjects or selected cohort of non-patients are potentially at high risk of bias. Third, some clinical observations should be made with respect to the possible interpretation of the described weak associations. Indeed, despite the fact that dental literature has predominantly been directed towards the view of dental occlusion as the cause of TMDs, the inverse relationship may even be more plausible and should have been considered to explain the occasionally described association between cross-sectionally observed phenomena. For instance, the association between unilateral cross-bite and TMJ disorders, which was described in three studies, has been recently shown to be independent on the correction of cross-bite (47). This means that in patients with TMJ disorders, the presence of cross-bite is not causative of the joint pathology, but it could be even viewed as the consequence of a certain skeletal morphology. A similar conclusion can be reached in the case of sagittal skeletal profiles that are associated with an increased risk for disc displacement (78). Such suggestion is in line with recent observations that orthodontics is neutral as far as the temporomandibular disorders are concerned (16). Similar suggestions have been proposed also for the purported relationship between anterior open bite and TMJ osteoarthrosis, with the former being the consequence, rather than the cause, of the latter (59). Moreover, the findings of a higher prevalence of CRMI slide and functional interferences in TMD patients, as reported by a few papers $(57,66)$, can be explained with the pain-related adaptation of motor functioning, rather than considered the cause of pain (79-81).

In summary, it can be concluded that some significant associations between occlusal variables and TMD have been occasionally described, but they are not consistent across studies (i.e., reported in most researches). Alternative explanations for the presence of such features in TMD patients with respect to their purported causal role (e.g., consequence of peculiar skeletal anatomy or TMJ disease) tended to be ignored by the dental communities over the past few 
Table 4. Summary of findings of studies adopting multiple variable analysis: number of papers reporting the presence and absence of an association with TMD per each of the most frequently investigated occlusal feature. For positive associations, reported OR and the TMD category are put in parentheses

\begin{tabular}{|c|c|c|}
\hline Occlusal features & Non-patient studies & Patient studies \\
\hline \multirow[t]{2}{*}{ Overjet } & Association: $N=0$ & Association $N=1$ (OR $2 \cdot 83$ for TMJ pain) \\
\hline & No association: $N=2$ & No association $N=8$ \\
\hline \multirow[t]{2}{*}{ Overbite } & Association: $N=0$ & Association: $N=0$ \\
\hline & No association: $N=2$ & No association: $N=10$ \\
\hline \multirow[t]{2}{*}{ Open Bite } & $\begin{array}{l}\text { Association: } N=2 \\
\text { (anterior open bite OR } \\
3 \cdot 6 \text { for myofascial pain; } \\
\text { posterior open bite OR } 4 \cdot 0 \text { for TMD) }\end{array}$ & Association: $N=1$ (OR $7 \cdot 27$ for osteoarthrosis) \\
\hline & No association: $N=1$ & No association: $N=8$ \\
\hline \multirow[t]{2}{*}{ Unilateral Cross-Bite } & Association: $N=0$ & $\begin{array}{l}\text { Association: } N=3 \text { (OR } 3.33 \text { for DDR, } \\
\text { OR } 2.64 \text { for DDNR, OR } 11.67 \text { for intra-capsular TMD) }\end{array}$ \\
\hline & No association: $N=1$ & No association: $N=6$ \\
\hline \multirow[t]{2}{*}{ CR-MI Slide } & Association: $N=0$ & Association: $N=1$ (OR 2.57 for myofascial pain) \\
\hline & No association: $N=1$ & No association: $N=8$ \\
\hline \multirow[t]{2}{*}{ Midline Discrepancy } & Association: $N=0$ & Association: $N=0$ \\
\hline & No association: $N=4$ & No association: $N=8$ \\
\hline \multirow[t]{2}{*}{ Posterior Missing Teeth } & $\begin{array}{l}\text { Association: } N=1 \\
\text { (OR } 4 \cdot 2 \text { for myofascial pain) }\end{array}$ & Association: $N=0$ \\
\hline & No association: $N=1$ & No association: $N=8$ \\
\hline \multirow[t]{2}{*}{ Molar Class } & Association: $N=0$ & Association: $N=0$ \\
\hline & No association: $N=1$ & No association: $N=5$ \\
\hline \multirow[t]{2}{*}{ Molar Asymmetry } & - & Association: $N=0$ \\
\hline & & No association: $N=5$ \\
\hline \multirow[t]{2}{*}{ Mediotrusive Interferences } & Association: $N=0$ & $\begin{array}{l}\text { Association: } N=2 \text { (OR } 2.45 \text { for myofascial pain; } \\
\text { OR } 2 \cdot 14 \text { for disc displacement) }\end{array}$ \\
\hline & No association: $N=1$ & No association: $N=1$ \\
\hline \multirow[t]{2}{*}{ Laterotrusive Interferences } & Association: $N=0$ & Association: $N=1$ (OR $2 \cdot 67$ for TMJ pain) \\
\hline & No association: $N=1$ & No association: $N=2$ \\
\hline
\end{tabular}

OR, odds ratio; TMJ, temporomandibular joint; DDR, disc displacement with reduction; DDNR, disc displacement without reduction.

decades $(3,7)$. In addition, epidemiologic studies of dental occlusion have demonstrated that purported malocclusions and occlusal dysharmonies should be viewed as ancillary findings that are also present with the same frequency in non-TMD patients (82). Thus, even the pre-requisite to hypothesise a causal role for dental occlusion in TMD patients, viz., the presence of a strong and consistent association between the two phenomena (i.e., occlusal feature and TMD), is lacking. On the contrary, the literature is strong and consistent to support the role of other factors, such as psychosocial and genetic issues as well as musclerelated overload, in the pathophysiology of temporomandibular disorders $(1,2,83)$.

Such observations should ideally lead to an end of the so-called 'gnathological era' of aetiological thinking in the TMD field, in which normal variability in the interindividual features of dental occlusion has been considered a pathological sign. Based on this suggestion, future teaching about these topics for the dental specialties working on the correction of dental occlusion should be introduced in their academic training as well as in their clinical practices.

\section{Conclusions}

This manuscript reviewed the literature on the association between features of dental occlusion and temporomandibular disorders. Based on findings, which support the absence of a disease-specific association, there is no ground to hypothesise a major role for dental occlusion in the pathophysiology of TMDs. Dental clinicians are thus encouraged to move forward and abandon the old-fashioned gnathological paradigm. 


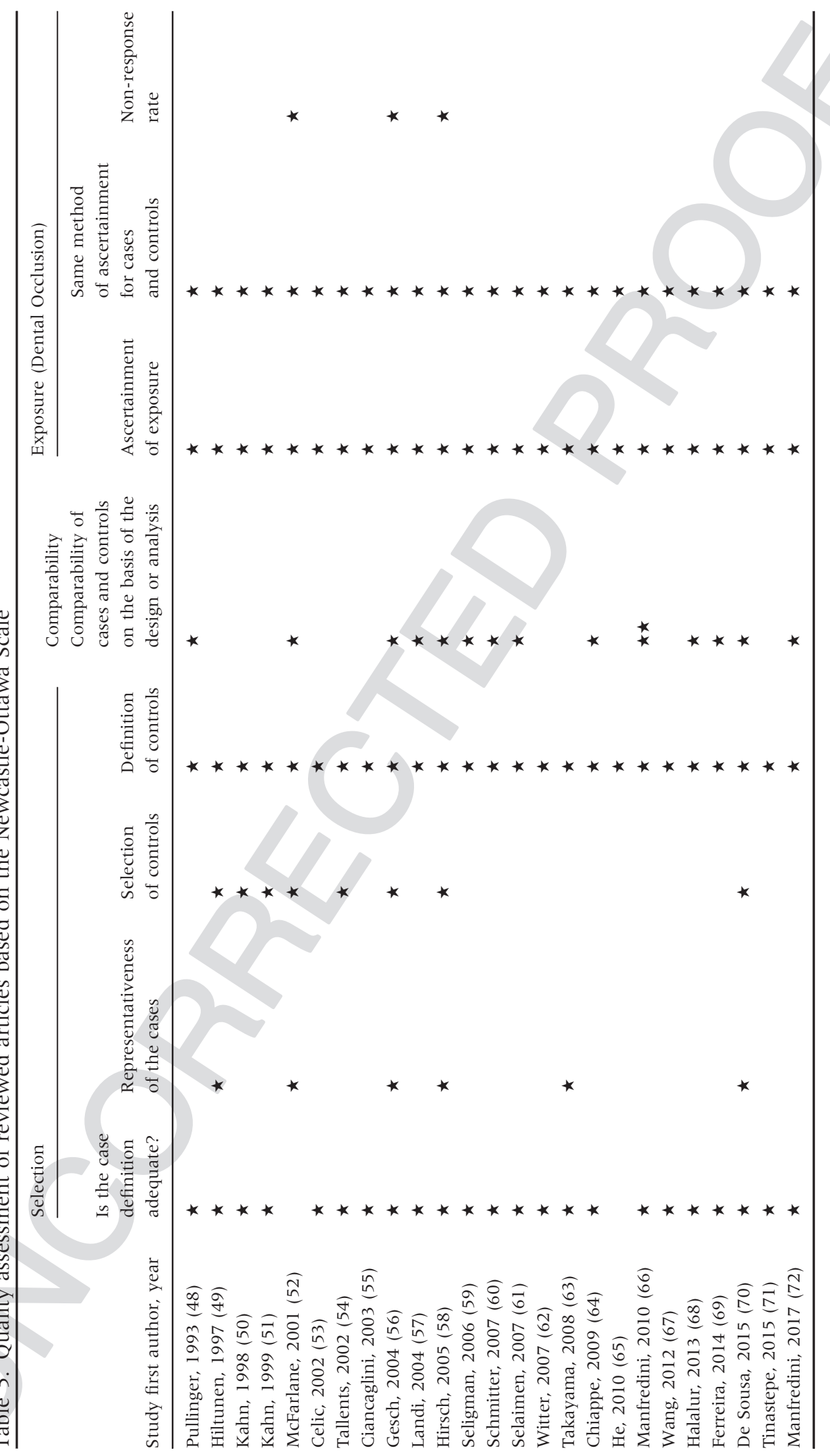




\section{Conflicts of interest}

The authors have stated explicitly that there are no conflicts of interest in connection with this article.

\section{Funding}

The authors did not receive any funding to prepare this manuscript.

\section{References}

1. Suvinen TI, Kemppainen P, Könönen M, Dworkin SF. Review of aetiological concepts of temporomandibular pain disorders: towards a biopsychosocial model for integration of physical disorder factors with psychological and psychosocial illness impact factors. Eur J Pain. 2005;9:613-633.

2. Slade GD, Ohrbach R, Greenspan JD, Fillingim RB, Bair E, Sanders AE et al. Painful temporomandibular disorder: decade of discovery from OPPERA studies. J Dent Res. 2016;95:1084-1092.

3. Okeson JP. Evolution of occlusion and temporomandibular disorder in orthodontics: past, present, and future. Am J Orthod Dentofacial Orthop. 2015;147(5 Suppl):S216-S223.

4. deLeeuw R, Klasser GD. The American Academy of Orofacial Pain. Orofacial pain: guidelines for assessment, diagnosis, and management. Chicago: Quintessence Publishing; 2013.

5. Manfredini D, Guarda-Nardini L, Winocur E, Piccotti F, Ahlberg J, Lobbezoo F. Research diagnostic criteria for temporomandibular disorders. A systematic review of axis I epidemiological findings. Oral Surg Oral Med Oral Pathol Oral Radiol Endod. 2011;112:453-462.

6. Manfredini D, Ahlberg J, Winocur E, Guarda-Nardini L, Lobbezoo F. Correlation of RDC/TMD axis I diagnoses and axis II pain-related disability. A multicenter study. Clin Oral Investig. 2011;15:749-756.

7. Türp JC, Greene CS, Strub JR. Dental occlusion: a critical reflection on past, present and future concepts. J Oral Rehabil. 2008;35:446-453.

8. Ash MM. Paradigmatic shifts in occlusion and temporomandibular disorders. J Oral Rehabil. 2001;28:1-13.

9. Greene CS. The etiology of temporomandibular disorders: implications for treatment. J Orofac Pain. 2001;15:93-105.

10. Manfredini D, Favero L, Gregorini G, Cocilovo F, GuardaNardini L. Natural course of temporomandibular disorders with low pain-related impairment: a 2-to-3-year follow up study. J Oral Rehabil. 2013;40:436-442.

11. Schwartz RA, Greene CS, Laskin DM. Personality characteristics of patients with myofascial pain-dysfunction (MPD) syndrome unresponsive to conventional therapy. J Dent Res. 1979;58:1435-1439.

12. Kotiranta U, Suvinen T, Kauko T, Le Bell Y, Kemppainen P, Suni $\mathrm{J}$ et al. Subtyping patients with temporomandibular disorders in a primary health care setting on the basis of the research diagnostic criteria for temporomandibular disorders axis II pain-related disability: a step toward tailored treatment planning? J Oral Facial Pain Headache. 2015;29:126134.

13. Gustin SM, Burke LA, Peck CC, Murray GM, Henderson LA. Pain and personality: do individuals with different forms of chronic pain exhibit a mutual personality? Pain Pract. 2016;16:486-494.

14. Koh H, Robinson PG. Occlusal adjustment for treating and preventing temporomandibular joint disorders. Cochrane Database Syst Rev 2003:CD003812.

15. Luther F, Layton S, McDonald F. Orthodontics for treating temporomandibular joint (TMJ) disorders. Cochrane Database Syst Rev 2010:CD006541. https://doi.org/10.1002/ 14651858.cd006541.pub2

16. Manfredini D, Stellini E, Gracco A, Lombardo L, Nardini LG, Siciliani G. Orthodontics is temporomandibular disordersneutral. Angle Orthod. 2016;89:649-654.

17. Türp JC, Schindler H. The dental occlusion as a suspected cause for TMDs: epidemiological and etiological considerations. J Oral Rehabil. 2012;39:502-512.

18. Manfredini D, Castroflorio T, Perinetti G, Guarda-Nardini L. Dental occlusion, body posture, and temporomandibular disorders: where we are now and where we are heading for. J Oral Rehabil. 2012;39:463-471.

19. Pullinger A. Establishing better biological models to understand occlusion. I: TM joint anatomic relationships. J Oral Rehabil. 2013;40:296-318.

20. Alanen P. Occlusion and temporomandibular disorders (TMD): still unsolved question? J Dent Res. 2002;81:518519.

21. Slavicek R. Relationship between occlusion and temporomandibular disorders: implications for the gnathologist. Am J Orthod Dentofacial Orthop. 2011;139:10, 12, 14 passim.

22. Sackett DL, Rosenberg WM, Gray JA, Haynes RB, Richardson WS. Evidence based medicine: what it is and what it isn't. BMJ. 1996;312:71-72.

23. Egermark-Eriksson I, Carlsson GE, Magnusson T. A long-term epidemiologic study of the relationship between occlusal factors and mandibular dysfunction in children and adolescents. J Dent Res. 1987;66:67-71.

24. Runge ME, Sadowsky C, Sakols EI, BeGole EA. The relationship between temporomandibular joint sounds and malocclusion. Am J Orthod Dentofacial Orthop. 1989 Jul;96:36-42.

25. Al-Hadi ????. Prevalence of temporomandibular disorders in relation to some occlusal parameters. J Prosthet Dent. 1993;70:345-350.

26. Christensen LV, Donegan SJ, McKay DC. Mediotrusive tooth guidance and temporomandibular joint sounds in non-patients and patients. J Oral Rehabil. 1996;23:686-698.

27. Donegan SJ, Christensen LV, McKay DC. Canine tooth guidance and temporomandibular joint sounds in nonpatients and patients. J Oral Rehabil. 1996;23:799-804.

28. Liu JK, Tsai MY. Relationship between morphologic malocclusion and temporomandibular disorders in orthodontic patients prior to treatment. Funct Orthod. 1997;14:13-16. 
29. Minagi S, Ohtsuki H, Sato T, Ishii A. Effect of balancing-side occlusion on the ipsilateral TMJ dynamics under clanging. J Oral Rehabil. 1997;24:57-62.

30. Ciancaglini R, Gherlone EF, Radaelli G. Association between loss of occlusal support and symptoms of functional disturbances of the masticatory system. J Oral Rehabil. 1999;26:248-253.

31. Pullinger AG, Seligman DA. Quantification and validation of predictive values of occlusal variables in temporomandibular disorders using a multifactorial analysis. J Prosthet Dent. 2000;83:66-75.

32. John MT, Hirsch C, Drangsholt MT, Mancl LA, Setz JM. Overbite and overjet are not related to self-report of temporomandibular disorder symptoms. J Dent Res. 2002;81:164-169.

33. Fuji T. The relationship between the occlusal interference side and the symptomatic side in temporomandibular disorders. J Oral Rehabil. 2003;30:295-300.

34. Sarita PT, Kreulen CM, Witter D, Creugers NH. Signs and symptoms associated with TMD in adults with shortened dental arches. Int J Prosthodont. 2003;16:265-270.

35. Pahkala R, Qvarnström M. Can temporomandibular dysfunction signs be predicted by early morphological or functional variables? Eur J Orthod. 2004 Aug;26:367-373.

36. Mundt T, Mack F, Schwahn C, Bernhardt O, Kocher T, John $\mathrm{U}$ et al. Gender differences in associations between occlusal support and signs of temporomandibular disorders: results of the population-based Study of Health in Pomerania (SHIP). Int J Prosthodont. 2005;18:232-239.

37. Sipilä K, Ensio K, Hanhela H, Zitting P, Pirttiniemi P, Raustia A. Occlusal characteristics in subjects with facial pain compared to a pain-free control group. Cranio. 2006;24:245-251.

38. Wang MQ, Cao HT, Liu FR, Chen C, Li G. Association of tightly locked occlusion with temporomandibular disorders. J Oral Rehabil. 2007;34:169-173.

39. Badel T, Marotti M, Krolo I, Kern J, Keros J. Occlusion in patients with temporomandibular joint anterior disk displacement. Acta Clin Croat. 2008;47:129-136.

40. Wang MQ, Xue F, He JJ, Chen JH, Chen CS, Raustia A. Missing posterior teeth and risk of temporomandibular disorders. J Dent Res. 2009;88:942-945.

41. Marklund S, Wänman A. Risk factors associated with incidence and persistence of signs and symptoms of temporomandibular disorders. Acta Odontol Scand. 2010;68:289-299.

42. Lauriti L, Motta LJ, Silva PF, Leal de Godoy CH, Alfaya TA, Fernandes KP et al. Are occlusal characteristics, headache, parafunctional habits and clicking sounds associated with the signs and symptoms of temporomandibular disorder in adolescents? J Phys Ther Sci. 2013;25:1331-1334.

43. Manfredini D, Vano M, Peretta R, Guarda-Nardini L. Jaw clenching effects in relation to two extreme occlusal features: patterns of diagnoses in a TMD patient population. J Craniomand Sleep Pract. 2014;32:45-50.

44. Manfredini D, Stellini E, Marchese-Ragona R, Guarda-Nardini L. Are occlusal features associated with different temporomandibular disorder diagnoses in bruxers? J Craniomand Sleep Pract. 2014;32:283-288.

45. Manfredini D, Perinetti G, Guarda-Nardini L. Dental malocclusion is not related to temporomandibular joint clicking: a logistic regression analysis in a patient population. Angle Orthod. 2014;84:310-315.

46. Baldini A, Nota A, Cozza P. The association between occlusion time and temporomandibular disorders. J Electromyogr Kinesiol. 2015;25:151-154.

47. Michelotti A, Iodice G, Piergentili M, Farella M, Martina R. Incidence of temporomandibular joint clicking in adolescents with and without unilateral posterior cross-bite: a 10year follow-up study. J Oral Rehabil. 2016;43:16-22.

48. Pullinger AG, Seligman DA, Gornbein JA. A multiple logistic regression analysis of the risk and relative odds of temporomandibular disorders as a function of common occlusal features. J Dent Res. 1993;72:968-979.

49. Hiltunen K, Vehkalahti M, Ainamo A. Occlusal imbalance and temporomandibular disorders in the elderly. Acta Odontol Scand. 1997;55:137-141.

50. Kahn J, Tallents RH, Katzberg RW, Moss ME, Murphy WC. Association between dental occlusal variables and intraarticular temporomandibular joint disorders: horizontal and vertical overlap. J Prosthet Dent. 1998;79:658-662.

51. Kahn J, Tallents RH, Katzberg RW, Ross ME, Murphy WC. Prevalence of dental occlusal variables and intraarticular temporomandibular disorders: molar relationship, lateral guidance, and nonworking side contacts. J Prosthet Dent. 1999;82:410-415.

52. Macfarlane TV, Gray RJM, Kincey J, Worthington HV. Factors associated with the temporomandibular disorder, pain dysfunction syndrome (PDS): manchester case-control study. Oral Dis. 2001;7:321-330.

53. Celic R, Jerolimov V, Panduric J. A study of influence of occlusal factors and parafunctional habits on the prevalence of signs and symptoms of TMD. Int J Prosthod. 2002;15:43-48.

54. Tallents RH, Macher DJ, Kyrkanides S, Katzberg RW, Moss ME. Prevalence of missing posterior teeth and intraarticular temporomandibular disorders. J Prosthet Dent. 2002;87:4550.

55. Ciancaglini R, Gherlone EF, Radaelli G. Unilateral temporomandibular disorder and asymmetry of occlusal contacts. J Prosthet Dent. 2003;89:180-185.

56. Gesch D, Bernhardt O, Kocher T, John U, Hensel E, Alte D. Association of malocclusion and functional occlusion with signs of temporomandibular disorders in adults: results of the population-based study of health in Pomerania. Angle Orthod. 2004;74:512-520.

57. Landi N, Manfredini D, Tognini F, Romagnoli M, Bosco M. Quantification of the relative risk of multiple occlusal variables for muscle disorders of the stomatognathic system. J Prosthet Dent. 2004;92:190-195.

58. Hirsch C, John MT, Drangsholt MT, Mancl LA. Relationship between overbite/overjet and clicking or crepitus of the temporomandibular joint. J Orofac Pain. 2005;19:218-225.

59. Seligman DA, Pullinger AG. Dental attrition models predicting temporomandibular joint disease or masticatory muscle 
pain versus asymptomatic controls. J Oral Rehabil. 2006;33:789-799.

60. Schmitter M, Balke Z, Hassel A, Ohlmann B, Rammelsberg $P$. The prevalence of myofascial pain and its association with occlusal factors in a threshold country non-patient population. Clin Oral Investig. 2007;11:277-281.

61. Selaimen CM, Jeronymo JC, Brilhante DP, Lima EM, Grossi PK, Grossi ML. Occlusal risk factors for temporomandibular disorders. Angle Orthod. 2007;77:471-477.

62. Witter DJ, Kreulen CM, Mulder J, Creugers NH. Signs and symptoms related to temporomandibular disorders-Followup of subjects with shortened and complete dental arches. J Dent. 2007;35:521-527.

63. Takayama Y, Miura E, Yuasa M, Kobayashi K, Hosoi T. Comparison of occlusal condition and prevalence of bone change in the condyle of patients with and without temporomandibular disorders. Oral Surg Oral Med Oral Pathol Oral Radiol Endod. 2008;105:104-112.

64. Chiappe G, Fantoni F, Landi N, Biondi K, Bosco M. Clinical value of 12 occlusal features for the prediction of disc displacement with reduction (RDC/TMD Axis I group IIa). J Oral Rehabil. 2009;36:322-329.

65. He SS, Deng X, Wamalwa P, Chen S. Correlation between centric relation; maximum intercuspation discrepancy and temporomandibular joint dysfunction. Acta Odontol Scand. 2010;68:368-376.

66. Manfredini D, Peretta R, Guarda-Nardini L, Ferronato G. Predictive value of combined clinically diagnosed bruxism and occlusal features for TMJ pain. Cranio. 2010;28:105113.

67. Wang C, Yin X. Occlusal risk factors associated with temporomandibular disorders in young adults with normal occlusions. Oral Surg Oral Med Oral Pathol Oral Radiol. 2012;114:419-423.

68. Haralur SB. Digital evaluation of functional occlusion parameters and their association with temporomandibular disorders. J Clin Diagn Res. 2013;7:1772-1775.

69. Ferreira FM, Simamoto-Junior PC, Resende Novais V, Tavares M, Fernandes-Neto AJ. Correlation between temporomandibular disorders, occlusal factors, and oral parafunctions in undergraduate students. Braz J Oral Sci. 2014;13:281-287.

70. de Sousa ST, de Mello VV, Magalhães BG, de Assis Morais MP, Vasconcelos MM, de França Caldas Junior A et al. The role of occlusal factors on the occurrence of temporomandibular disorders. Cranio. 2015;33:211-216.

71. Tinastepe $\mathrm{N}$, Oral $\mathrm{K}$. Investigation of the relationship between increased vertical overlap with minimum horizontal overlap and the signs of temporomandibular disorders. J Prosthodont. 2015;24:463-468.

72. Manfredini D, Lombardo L, Siciliani G. Dental angle class asymmetry and temporomandibular disorders. J Orofac Orthop. 2017;???:???-??? (in press).

73. Dworkin SF, LeResche L. Research diagnostic criteria for temporomandibular disorders: review, criteria, examinations and specifications, critique. J Craniomandib Disord. 1992;6:301-355.

74. Perinetti G, Contardo L. Posturography as a diagnostic aid in dentistry: a systematic review. J Oral Rehabil. 2009;36:922-936.

75. Hill BA. The environment and disease: association or causation? Proceed Royal Soc Med. 1965;58:295-300.

76. Manfredini D, Bucci MB, Montagna F, Guarda-Nardini L. Temporomandibular disorders assessment: medicolegal considerations in the evidence-based era. J Oral Rehabil. 2011;38:101-119.

77. Reid KI, Greene CS. Diagnosis and treatment of temporomandibular disorders: an ethical analysis of current practices. J Oral Rehabil. 2013;40:546-561.

78. Manfredini D, Segù M, Arveda N, Lombardo L, Siciliani G, Rossi A et al. Temporomandibular joint disorders in patients with different facial morphology. A systematic review of the literature. J Oral Maxillofac Surg. 2016;74:29-46.

79. Lund JP, Donga R, Widmer CG, Stohler CS. The pain-adaptation model: a discussion of the relationship between chronic musculoskeletal pain and motor activity. Can J Physiol Pharmacol. 1991;69:683-694.

80. Murray GM, Peck CC. Orofacial pain and jaw muscle activity: a new model. J Orofac Pain. 2007;21:263-278.

81. Manfredini D, Cocilovo F, Stellini E, Favero L, Guarda-Nardini L. Surface electromyography findings in unilateral myofascial pain patients: comparison of painful vs non painful sides. Pain Med. 2013;14:1848-1853.

82. Manfredini D, Perinetti G, Stellini E, Di Leonardo B, Guarda-Nardini L. Prevalence of static and dynamic dental malocclusion features in subgroups of temporomandibular disorder patients: implications for the epidemiology of the TMD-occlusion association. Quintessence Int. 2015;46:341349.

83. Ohrbach R, Bair E, Fillingim RB, Gonzalez Y, Gordon SM, Lim PF et al. Clinical orofacial characteristics associated with risk of first-onset TMD: the OPPERA prospective cohort study. J Pain. 2013;14(12 Suppl):T33-T50.

Correspondence: Daniele Manfredini, Via Ingolstadt 3, 5400 Marina di Carrara (MS), Italy.

E-mail: daniele.manfredini@tin.it 